\title{
Capacitated Location and Allocation Models of Long-Term Care Facilities
}

\author{
Byung Duk Song \\ Department of Industrial and Systems Engineering, Korea Advanced Institute of Science and Technology, Daejeon, Korea \\ Young Dae Ko \\ Industrial Engineering and Management Research Institute, Korea Advanced Institute of Science and Technology, Daejeon, \\ James R. Morrison, Hark Hwang* \\ Department of Industrial and Systems Engineering, Korea Advanced Institute of Science and Technology, Daejeon, Korea
}

(Received: February 1, 2013 / Revised: June 4, 2013; August 27, 2013 / Accepted: September 6, 2013)

\begin{abstract}
People are living longer than ever before. As a result, life expectancy is going up and the demand of long-term care facilities is increasing in most countries. The facilities provide rehabilitative, restorative, and skilled nursing care to patients or residents in need of assistance with activities of daily living. This study deals with the capacitated location and allocation problem of long-term care facilities in a city that consists of a finite number of regions. Assuming that in each region candidate locations for three types of facilities are already given, two integer programming models are developed under the closest assignment rule reflecting the demand characteristics of the facilities. Both the location and type of the facilities to be built become decision variables. To show the validity of the models, numerical problems are solved with commercial software, CPLEX. Also, sensitivity studies were conducted to identify relationships between the system parameters.
\end{abstract}

Keywords: Long-Term Care Facility, Capacitated Location-Allocation Problem, Closest Assignment Rule, Three Types of Facilities

* Corresponding Author, E-mail: hhwang@kaist.ac.kr

\section{INTRODUCTION}

\subsection{Problem Description}

Due to the advancement of medicines and improvement in health care services, the average life expectancy has increased continuously in both developed and developing countries. Thus the population of aged people is rising steadily with the decreasing mortality rate and the number of patients stricken by the senile diseases, such as stroke and Alzheimer disease, is sharply increasing. For instance, according to the statistics of the Korean government, as of year 2009 more than 650,000 aged people were stricken with the age-related diseases. Thus, in aged society, the treatment of patients who have senile diseases becomes an important social issue and it has to be considered at the national level. Currently, many countries have made various supportive provisions for those patients but the existing public social infrastructures still do not satisfy the increasing demand. The coverage rate of the public long-term care facilities is only $4.0 \%$ in Korea, while that of Japan and Germany is $10.2 \%$ and $10.0 \%$, respectively. The Korean government intends to increase the ratio up to $30 \%$ by establishing additional public long-term care facilities. In general, the senile diseases patients and their families preferpublic facilities rather than private facilities mainly for economic reason, i.e., public facilities offer ser- 
vices at a significantly reduced fee. Moreover, they prefer facilities located close to their home for the patient's emotional stability and easy access to the family members. Motivated by these social necessities and demand characteristics, we want to determine locations and types of the public long-term care facilities to be newly built and also allocate patients when they are built. The problem we deal with in this paper is as follows. Suppose it is desired to establish long-term care facilities in a metropolitan area that consists of a finite number of regions. The health care demand data of each region are readily available in the form of estimates by the government. Let the demand center of a region be a point destination weighted by the health care demand of that region. For simplicity, the location of demand center in each region is assumed to be already known. Designing health care facilities requires the accommodation of conflicting demands from various constituencies, balancing costs and needs, and satisfying stringent regulatory and accreditation rules. Therefore, we assume that the government has already determined three types of design standards of long-term care facilities to provide economically safe and effective health care. They differ in terms of size, capacity and construction cost. With restriction on either the amount of budget for constructing the facilities or the maximum travelling distance allowed of the patients, we want to identify the regions where opening long-term care facilities is desirable as well as the types of facility, if decided to open. In allocating patients to the facilities, the closest-to-home assignment rule is imposed to reflect the demand characteristics of the facilities.

\subsection{Literature Review}

The problem in this paper is a type of the capacitated location-allocation problem. Capacitated facility location problem has been studied for several decades and many applications were reported in the literature. Dearing and Newruck (1979) suggested a capacitated facility location problem to meet customer demands economically. In the objective function, both the transportation cost and total cost of opening facilities were included and the Lagrangian relaxation method was adopted to solve the problem. Sankaran and Raghavan (1997) suggested a locating and sizing facilities problem of South India. They considered more than one type of facility size and used LINDO to solve the proposed model. Nozick (2001) proposed a capacitated facility location model with coverage restrictions. The objective was to minimize the fixed cost in locating facilities while maintaining an appropriate level of service. Single type facility was considered and two Lagrangian relaxation based heuristics were developed to solve the problem. Berman et al. (2006) analyzed the problem of lo- cating a set of service facilities on a network when the demand for service is stochastic. They developed a mathematical model to reduce the demand loss by finding the minimum number of facilities and their locations. A number of research papers on facility location problems in health care systems have been presented. Rahman and Smith (2000) and Daskin and Dean (2004) reported review articles, Adenso-Diaz and Rodriguez (1997) and Jia et al. (2007) dealt with the emergency medical service facilities, Verter and Lapierre (2002) studied preventive care facilities, and Ndiaye and Alfares (2008) wrote an article on healthcare facilities for seasonally moving populations. Recently, Kim and Kim (2010) applied closest allocation constraints for determining the locations of public long-term care facilities. They considered incapacitated long-term care facility of single type and tried to balance the numbers of patients assigned to the facilities. Kim et al. (2012) dealt with the problem of locating two types of public health-care facilities, public hospitals that provide both hospital services and homecare services and health centers that provide only homecare services. With the objective of minimizing the total construction cost, they proposed two types of heuristics, one based on priority rules and the other based on approximate mathematical formulation. In real world situation, the establishment cost of a facility is usually affected by land availability, facility size and unit land price. Thus we integrate the reality aspects in our mathematical model as follows: First, at most one facility can be built in a region among three types of facilities, i.e., small, medium, and large, each differing in capacity and construction cost. The objective adopted mostly in the previous researches is the minimization of some appropriate total cost function. There can be other criteria for a number of real-world layout and location problems. Thus mathematical models with different objective functions and constraints are developed in this paper, through which the relationships among the budget, inconvenience of the patients and the establishment cost are examined. The remainder of this paper is organized as follows: in the next section, the assumptions and notations of the problem are described and then two kinds of mathematical models are developed. The validity of the developed models is illustrated through various numerical problems and the results obtained by CPLEX are listed in Section 3. Section 4 concludes the paper with suggestions for further studies.

\section{MODEL DEVELOPMENT}

\subsection{Assumptions}

1) In each region at most one facility can be built among three types of facilities, small, medium, and large size. 
They are different in capacities and construction costs. Candidate locations for facilities are known.

2) It is assumed that demand data do not include those patients already receiving medical services from existing facilities. This study deals with the problem of building new facilities for the patients who do not receive proper medical treatment yet by answering the questions of where to build new facilities and how to allocate the patients to newly built facilities.

3) Let patient group $i$ denote all the patients residing in region $i$. Patient group $i$ dwells at appoint called demand center $i$ whose location is assumed to be already known.

4) The demand of each patient group is equal to or less than the capacity of large size facility.

5) Each patient group cannot be served by more than one facility. If a facility opens in region $i$, patient group $i$ has to be served by the facility regardless of the distance between the demand centre and location of the facility. If no facility opens in region $i$ patient group $i$ receive services from the closest facility.

\subsection{Notations}

$i, j \quad$ : indices of patient groups and candidate facility locations

$J \quad$ : index set of regions

$K \quad$ : index set of facility types

$a_{i} \quad$ : number of patients at region $i$

$d^{s}{ }_{i j} \quad$ : distance between the demand center and the candidate location $j$ of small size facility in region $i$

$d^{m}{ }_{i j} \quad$ : distance between the demand center and the candidate location $j$ of medium size facility in region $i$

$d_{i j}^{l} \quad$ : distance between the demand center and the candidate location $j$ of large size facility in region $i$

$F_{j}^{s} \quad$ : establishment cost of small size facility in region $j$

$F_{j}^{m} \quad$ : establishment cost of medium size facility in region $j$

$F_{j}^{l} \quad$ : establishment cost of large size facility in region i

$B \quad$ : amount of budget available for opening the facilities

$C^{S} \quad$ : capacity of small size facility (number of patients)

$C^{m} \quad$ : capacity of medium size facility (number of patients)

$C^{l} \quad$ : capacity of large size facility (number of patients)

$S_{\text {Max }}:$ upper limit on the maximum number of small facilities to open

$M_{\text {Max }}$ : upper limit on the maximum number of medium size facilities to open

$L_{\text {Max }}$ : upper limit on the maximum number of large size facilities to open

$D_{M A X}:$ maximum allowed average traveling distance

$y_{j}^{s} \quad$ : location decision variable. It will be equal to 1 if a small size facility will open in region $j$. Otherwise, 0 .

$y_{j}^{m} \quad$ : location decision variable. It will be equal to 1 if a medium size facility will open in region $j$. Otherwise, 0 .

$y_{j}^{l} \quad$ : location decision variable. It will be equal to 1 if a large size facility will open in region $j$. Otherwise, 0 .

$x_{i j}{ }^{s} \quad$ : allocation decision variable. It will be equal to 1 when patient group in region $i$ receives services from the small size facility opened in region $j$. Otherwise, 0 .

$x_{i j}{ }^{m} \quad$ : allocation decision variable. It will be equal to 1 when patient group in region $i$ receives services from the small size facility opened in region $j$. Otherwise, 0 .

$x_{i j}{ }^{l} \quad$ : allocation decision variable. It will be equal to 1 when patient group in region $i$ receives services from the large size facility opened in region $j$. Otherwise, 0 .

\subsection{Mathematical Models}

There could be more than one goal to achieve in the establishment of long-term care facilities. In this section, two mathematical models are developed, each with different objective function and constraints. The first model has the objective of minimizing the average travelling distance of the patients (ATDP) with the budget limit on the construction cost. In the second model, we find the minimum total establishment cost (TC) while satisfying the restriction on the maximum travelling distance (MTD) of patient group.

\subsubsection{Mathematical model 1}

$\operatorname{Min}\left(\sum_{i=1}^{J} \sum_{j=1}^{J} a_{i} d_{i j}^{s} x_{i j}^{s}+a_{i} d_{i j}^{m} x_{i j}^{m}+a_{i} d_{i j}^{l} x_{i j}^{l}\right) / \sum_{i=1}^{J} a_{i}$

Subject to

$$
\begin{array}{ll}
y_{j}^{s}+y_{j}^{m}+y_{j}^{l} \leq 1 & \forall j \in J \\
\sum_{i=1}^{J} x_{i j}^{k} \leq M \cdot y_{j}^{k} & \forall j \in J, k \in K \\
\sum_{j=1}^{J}\left(x_{i j}^{s}+x_{i j}^{m}+x_{i j}^{l}\right)=1 & \forall i \in J \\
\sum_{j=1}^{J}\left(d_{i j}^{s} x_{i j}^{s}+d_{i j}^{m} x_{i j}^{m}+d_{i j}^{l} x_{i j}^{l}\right) \leq d_{i j}^{k}+M\left(1-y_{j}^{k}\right) & \forall i, j \in J, k \in K \\
x_{j j}^{k}=y_{j}^{k} & \forall j \in J, k \in K
\end{array}
$$




$$
\begin{aligned}
& \sum_{j=1}^{J} y_{j}^{s} \leq S_{M a x} \\
& \sum_{j=1}^{J} y_{j}^{m} \leq M_{M a x} \\
& \sum_{j=1}^{J} y_{j}^{l} \leq L_{M a x} \\
& \sum_{i=1}^{J} a_{i}\left(x_{i j}^{s}+x_{i j}^{m}+x_{i j}^{l}\right) \leq C^{s} y_{j}^{s}+C^{m} y_{j}^{m}+C y_{j}^{l} \quad \forall j \in J \\
& \sum_{j=1}^{J} F_{j}^{s} y_{j}^{s}+F_{j}^{m} y_{j}^{m}+F_{j}^{l} y_{j}^{l} \leq B \\
& y_{j}^{s}, y_{j}^{m}, y_{j}^{l} \in\{0,1\} \quad \forall j \in J \\
& x_{i j}^{s}, x_{i j}^{m}, x_{i j}^{l}, \in\{0,1\} \\
&
\end{aligned}
$$

Eq. (1) denotes the objective function of minimizing the ATDP. Constraint (2) ensures that at most one facility can be established in each region. Constraint (3) expresses that patient group $i$ can be assigned to facility in region $j$ only when it opens. Also, all the patients belonging to patient group $i$ have to be assigned to a same facility by constraint (4). Constraint (5) enforces that each patient group has to be assigned to a closest open facility. Note that if a facility opens at candidate location in region $i$, patient group $i$ has to be served by the facility regardless of the distance between the centre point of the patient group and location of the facility. Thus, constraint (6) ensures that patient group gets medical services from its own region if any type of facility will open in the region. Constraints (7), (8), and (9) restrict the number of facilities of each type of facilities. Through these constraint sets, we prevent the situation that certain design of facility dominates. Constraint (10) represents the capacity of facilities. The total number of assigned patients at each facility cannot exceed the capacity of the facility. By constraint (11) the TC cannot exceed a given budget $(B)$. Constraints (12) and (13) denote the binary decision variable of our problem.

\subsubsection{Mathematical model 2}

$\operatorname{Min} \quad \sum_{j=1}^{J} F_{j}^{s} y_{j}^{s}+F_{j}^{m} y_{j}^{m}+F_{j}^{l} y_{j}^{l}$

Subject to

$$
\begin{array}{cc}
y_{j}^{s}+y_{j}^{m}+y_{j}^{l} \leq 1 & \forall j \in J \\
\sum_{i=1}^{J} x_{i j}^{k} \leq M \cdot y_{j}^{k} & \forall j \in J, k \in K
\end{array}
$$

$$
\begin{array}{ll}
\sum_{j=1}^{J}\left(x_{i j}^{s}+x_{i j}^{m}+x_{i j}^{l}\right)=1 & \forall i \in J \\
\sum_{j=1}^{J}\left(d_{i j}^{s} x_{i j}^{s}+d_{i j}^{m} x_{i j}^{m}+d_{i j}^{l} x_{i j}^{l}\right) \leq d_{i j}^{k}+M\left(1-y_{j}^{k}\right) & \forall i, j \in J, k \in K \\
x_{j j}^{k}=y_{j}^{k} & \forall j \in J, k \in K \\
\sum_{j=1}^{J} y_{j}^{s} \leq S_{M a x} & \\
\sum_{j=1}^{J} y_{j}^{m} \leq M_{M a x} & \\
\sum_{j=1}^{J} y_{j}^{l} \leq L_{M a x} & \\
\sum_{i=1}^{J} a_{i}\left(x_{i j}^{s}+x_{i j}^{m}+x_{i j}^{l}\right) \leq C^{s} y_{j}^{s}+C^{n} y_{j}^{m}+C y_{j}^{l} & \\
\max _{i \in J}\left\{d_{i j}^{s} x_{i j}^{s}+d_{i j}^{m} x_{i j}^{m}+d_{i j}^{l} x_{i j}^{l}\right\} \leq D_{M A X} & \forall j \in J \\
y_{j}^{s}, y_{j}^{m}, y_{j}^{l} \in\{0,1\} & \forall j \in J \\
x_{i j}^{s}, x_{i j}^{m}, x_{i j}^{l}, \in\{0,1\} & \forall i, j \in J
\end{array}
$$

In mathematical model 2 , the TC is minimized via the objective function (14). In order to guarantee an appropriate level of travelling distance of patients, the MTD allowed is restricted by constraint (15).

\section{NUMERICAL EXAMPLES}

The proposed model and algorithm were tested on the hypothetical data of 25 boroughs (regions) in the Seoul metropolitan city. The number of patients in each borough is based on the data from the Statistics Korea. It was obtained by multiplying the number of people aged over 65 of each borough and the average ratio of the senile disease incidence together. Let DMU and MU be the distance measurement unit and monetary unit, respectively. Two coordinates of the demand center were scaled on 2-dimensional plane with the range between $(0,0)$ and $(100,100)$ in DMU proportionally. For the candidate location of each type of facility, two coordinates $(x, y)$ were randomly generated from an interval of $(15,30)$ in DMU and added to the coordinate information of demand center. The opening costs in MU of small, medium and large size facilities in each borough were also randomly generated from intervals determined in advance. Table 1 shows for each region the number of patients, location coordinates of demand center, opening costs for small, medium and large size facilities, and candidate locations.

For instance, region 5 has 357 patients with its demand center location of $(19.2,33.0)$ in DMU. Distance 
Table 1. Hypothetical data for 25 boroughs in Seoul

\begin{tabular}{|c|c|c|c|c|}
\hline \multirow{2}{*}{$\begin{array}{l}\text { Re- } \\
\text { gion }\end{array}$} & $\begin{array}{l}\text { Demand } \\
\text { (patients) }\end{array}$ & $\begin{array}{l}\text { Small } \\
\text { (MU) }\end{array}$ & $\begin{array}{l}\text { Medium } \\
\text { (MU) }\end{array}$ & $\begin{array}{l}\text { Large } \\
\text { (MU) }\end{array}$ \\
\hline & $(\mathrm{x}, \mathrm{y})$ & $(\mathrm{x}, \mathrm{y})$ & $(\mathrm{x}, \mathrm{y})$ & $(\mathrm{x}, \mathrm{y})$ \\
\hline 1 & $\begin{array}{c}411 \\
(66.9,45.9)\end{array}$ & $\begin{array}{c}40 \\
(68.7,48.8)\end{array}$ & $\begin{array}{c}71 \\
(69.9,48.0)\end{array}$ & $\begin{array}{c}121 \\
(69.9,48.3)\end{array}$ \\
\hline 2 & $\begin{array}{c}376 \\
(72.0,21.0)\end{array}$ & $\begin{array}{c}34 \\
(73.7,23.1)\end{array}$ & $\begin{array}{c}76 \\
(73.6,23.0)\end{array}$ & $\begin{array}{c}114 \\
(74.9,23.5)\end{array}$ \\
\hline 3 & $\begin{array}{c}368 \\
(54.0,42.6)\end{array}$ & $\begin{array}{c}50 \\
(56.3,45.2)\end{array}$ & $\begin{array}{c}73 \\
(56.1,45.5)\end{array}$ & $\begin{array}{c}121 \\
(56.3,45.2)\end{array}$ \\
\hline 4 & $\begin{array}{c}364 \\
(40.2,48.3)\end{array}$ & $\begin{array}{c}41 \\
(42.5,50.9)\end{array}$ & $\begin{array}{c}88 \\
(42.7,49.8)\end{array}$ & $\begin{array}{c}129 \\
(43.1,51.0)\end{array}$ \\
\hline 5 & $\begin{array}{c}357 \\
(19.2,33.0)\end{array}$ & $\begin{array}{c}49 \\
(20.7,35.6)\end{array}$ & $\begin{array}{c}74 \\
(22.2,35.2)\end{array}$ & $\begin{array}{c}103 \\
(21.6,34.7)\end{array}$ \\
\hline 6 & $\begin{array}{c}349 \\
(41.7,9.90)\end{array}$ & $\begin{array}{c}37 \\
(44.3,11.4)\end{array}$ & $\begin{array}{c}61 \\
(43.2,12.0)\end{array}$ & $\begin{array}{c}105 \\
(44.1,11.7)\end{array}$ \\
\hline 7 & $\begin{array}{c}311 \\
(60.6,18.9)\end{array}$ & $\begin{array}{c}30 \\
(62.6,21.2)\end{array}$ & $\begin{array}{c}86 \\
(63.0,21.7)\end{array}$ & $\begin{array}{c}125 \\
(62.8,20.8)\end{array}$ \\
\hline 8 & $\begin{array}{c}297 \\
(42.3,22.5)\end{array}$ & $\begin{array}{c}43 \\
(43.8,24.5)\end{array}$ & $\begin{array}{c}79 \\
(45.0,25.5)\end{array}$ & $\begin{array}{c}119 \\
(45.3,24.2)\end{array}$ \\
\hline 9 & $\begin{array}{c}295 \\
(35.1,25.2)\end{array}$ & $\begin{array}{c}38 \\
(37.0,27.4)\end{array}$ & $\begin{array}{c}80 \\
(37.1,26.8)\end{array}$ & $\begin{array}{c}115 \\
(37.9,27.9)\end{array}$ \\
\hline 10 & $\begin{array}{c}294 \\
(62.7,36.0)\end{array}$ & $\begin{array}{c}48 \\
(65.2,39.0)\end{array}$ & $\begin{array}{c}69 \\
(64.4,38.8)\end{array}$ & $\begin{array}{c}115 \\
(64.8,38.8)\end{array}$ \\
\hline 11 & $\begin{array}{c}292 \\
(70.2,42.6)\end{array}$ & $\begin{array}{c}31 \\
(71.8,45.1)\end{array}$ & $\begin{array}{c}70 \\
(72.0,44.1)\end{array}$ & $\begin{array}{c}116 \\
(72.8,44.8)\end{array}$ \\
\hline 12 & $\begin{array}{c}288 \\
(53.4,46.8)\end{array}$ & $\begin{array}{c}30 \\
(55.1,49.1)\end{array}$ & $\begin{array}{c}70 \\
(56.0,49.3)\end{array}$ & $\begin{array}{c}115 \\
(55.7,48.9)\end{array}$ \\
\hline 13 & $\begin{array}{c}285 \\
(36.6,30.6)\end{array}$ & $\begin{array}{c}50 \\
(38.3,33.5)\end{array}$ & $\begin{array}{c}88 \\
(38.6,33.2)\end{array}$ & $\begin{array}{c}125 \\
(38.2,33.4)\end{array}$ \\
\hline 14 & $\begin{array}{c}279 \\
(38.4,36.0)\end{array}$ & $\begin{array}{c}39 \\
(41.4,38.8)\end{array}$ & $\begin{array}{c}63 \\
(40.4,37.9)\end{array}$ & $\begin{array}{c}121 \\
(39.9,38.1)\end{array}$ \\
\hline 15 & $\begin{array}{c}279 \\
(24.024 .3)\end{array}$ & $\begin{array}{c}31 \\
(26.5,26.6)\end{array}$ & $\begin{array}{c}82 \\
(26.3,26.8)\end{array}$ & $\begin{array}{c}129 \\
(26.0,26.7)\end{array}$ \\
\hline 16 & $\begin{array}{c}276 \\
(79.2,31.5)\end{array}$ & $\begin{array}{c}39 \\
(81.0,34.2)\end{array}$ & $\begin{array}{c}67 \\
(81.9,34.4)\end{array}$ & $\begin{array}{c}129 \\
(81.5,34.0)\end{array}$ \\
\hline 17 & $\begin{array}{c}272 \\
(21.6,18.6)\end{array}$ & $\begin{array}{c}47 \\
(23.2,21.2)\end{array}$ & $\begin{array}{c}61 \\
(23.5,21.1)\end{array}$ & $\begin{array}{c}121 \\
(24.5,21.4)\end{array}$ \\
\hline 18 & $\begin{array}{c}271 \\
(55.2,55.8)\end{array}$ & $\begin{array}{c}50 \\
(58.0,57.8)\end{array}$ & $\begin{array}{c}87 \\
(56.9,57.3)\end{array}$ & $\begin{array}{c}126 \\
(58.0,58.3)\end{array}$ \\
\hline 19 & $\begin{array}{c}256 \\
(52.8,15.0)\end{array}$ & $\begin{array}{c}49 \\
(55.8,17.2)\end{array}$ & $\begin{array}{c}65 \\
(54.4,17.7)\end{array}$ & $\begin{array}{c}118 \\
(55.1,17.0)\end{array}$ \\
\hline 20 & $\begin{array}{c}225 \\
(58.5,27.9)\end{array}$ & $\begin{array}{c}50 \\
(60.5,30.1)\end{array}$ & $\begin{array}{c}70 \\
(61.4,30.5)\end{array}$ & $\begin{array}{c}123 \\
(60.2,29.8)\end{array}$ \\
\hline 21 & $\begin{array}{c}224 \\
(69.0,27.0)\end{array}$ & $\begin{array}{c}44 \\
(71.1,28.8)\end{array}$ & $\begin{array}{c}67 \\
(70.6,28.6)\end{array}$ & $\begin{array}{c}112 \\
(70.5,29.9)\end{array}$ \\
\hline 22 & $\begin{array}{c}209 \\
(49.8,26.4)\end{array}$ & $\begin{array}{c}40 \\
(51.6,28.7)\end{array}$ & $\begin{array}{c}60 \\
(52.1,28.6)\end{array}$ & $\begin{array}{c}120 \\
(51.7,28.8)\end{array}$ \\
\hline 23 & $\begin{array}{c}165 \\
(30.9,11.4)\end{array}$ & $\begin{array}{c}34 \\
(32.5,13.8)\end{array}$ & $\begin{array}{c}61 \\
(33.9,14.3)\end{array}$ & $\begin{array}{c}115 \\
(33.3,13.1)\end{array}$ \\
\hline 24 & $\begin{array}{c}155 \\
(46.2,38.7)\end{array}$ & $\begin{array}{c}35 \\
(48.7,41.5)\end{array}$ & $\begin{array}{c}60 \\
(48.2,40.5)\end{array}$ & $\begin{array}{c}110 \\
(48.1,41.4)\end{array}$ \\
\hline 25 & $\begin{array}{c}118 \\
(52.5,33.6)\end{array}$ & $\begin{array}{c}46 \\
(54.3,35.4) \\
\end{array}$ & $\begin{array}{c}67 \\
(54.7,36.2) \\
\end{array}$ & $\begin{array}{c}117 \\
(54.7,35.8)\end{array}$ \\
\hline
\end{tabular}

MU: monetary unit. between demand center and candidate facility location was measured in Euclidean distances. For the facility capacities small, medium and large size are set at 300 , 500, and 700, respectively. Upper limit on the maximum number of each facility, $\left(S_{\operatorname{Max}}, M_{M a x}, L_{M a x}\right)$ was set to $(9,9$, 9 ). Let $J$ and $K$ be the number of regions and facility types, respectively. For instance, region 5 has 357 patients with its demand center location of $(19.2,33.0)$ in DMU. Distance between demand center and candidate facility location was measured in Euclidean distances. For the facility capacities small, medium and large size are set at 300,500 , and 700, respectively. Upper limit on the maximum number of each facility, $\left(S_{\operatorname{Max}}, M_{M a x}, L_{M a x}\right)$ was set to $(9,9,9)$. Let $\mathrm{J}$ and $\mathrm{K}$ be the number of regions and facility types, respectively.

Table 2 shows the number of decision variables and constraints in the proposed mathematical models. Thus with $\mathrm{J}=25$ and $\mathrm{K}=3$ in the above example problem, model 1 has 1,950 decision variables and 2,104 constraints while model 2 has 2,128 constraints.

To solve the models, we adopted CPLEX 12.1 which uses a branch and cut approach to find an optimal solution for problems that contain integer variables.

\subsection{Budget Restriction vs. ATDP}

Firstly, through mathematical model 1, we examined the relationship between budget restriction and ATDP. Table 3 shows the computational results with 9 different

Table 2. Number of decision variables and constraints in the proposed models

\begin{tabular}{lcc}
\hline & Model 1 & Model 2 \\
\hline No. of decision variables & $\mathrm{J} \cdot \mathrm{K}+\mathrm{J}^{2} \cdot \mathrm{K}$ & $\mathrm{J} \cdot \mathrm{K}+\mathrm{J}^{2} \cdot \mathrm{K}$ \\
No. of constraints & $\mathrm{J}(\mathrm{J} \cdot \mathrm{K}+2 \mathrm{~K}+3)+4$ & $\mathrm{~J}(\mathrm{~J} \cdot \mathrm{K}+2 \mathrm{~K}+4)+3$ \\
\hline
\end{tabular}

Table 3. Computational results of mathematical model 1

\begin{tabular}{cccc}
\hline Budget (MU) & ATDP (DMU) & TC (MU) & CPU time (s) \\
\hline 1,400 & 3.142 & 1,396 & 1.57 \\
1,450 & 3.083 & 1,446 & 1.22 \\
1,500 & 3.012 & 1,498 & 0.03 \\
1,550 & 2.978 & 1,537 & 0.84 \\
1,600 & 2.952 & 1,576 & 1.36 \\
1,650 & 2.921 & 1,631 & 0.95 \\
1,700 & 2.915 & 1,700 & 1.04 \\
1,750 & 2.896 & 1,737 & 0.03 \\
1,800 & 2.888 & 1,780 & 0.78 \\
\hline
\end{tabular}

ATDP: average travelling distance of the patients, TC: total establishment cost, MU: monetary unit, DMU: distance measurement unit. 


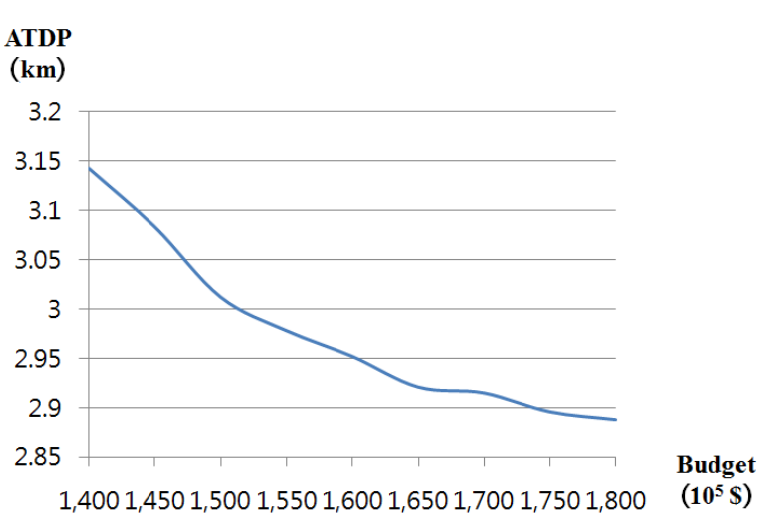

Figure 1. Relationship between average travelling distance of the patients (ATDP) and budget.

budget levels in MU.

Note that CPLEX took less than a few seconds of computation time in finding an optimal solution.

As the amount of budget increases, the ATDP decreases and that is cons is tent with our expectation. However, a substantial increase of the budget does not guarantee a corresponding decrease of ATDP as can be seen in Figure 1. While budget is increased by $28.57 \%$ from 1,400 to 1,800 , the ATDP decreases by only $8.08 \%$. The above observation needs to be considered for efficient use of budget.

\subsection{TC vs. $D_{\text {MAX }}$}

Secondly, the effects of maximum allowed travelling distance on the TC were studied. Table 4 shows the computational results of CPLEX. It can be observed that too strict restriction on the maximum allowed travelling distance makes the problem infeasible.

As the $D_{M A X}$ restriction is relaxed, the TC tends to decrease. However, when $D_{M A X}$ restriction is relaxed from 6.5 to 8.0, there is no saving in TC. Figure 2 shows the relationship between $D_{M A X}$ and TC. Under strict

Table 4. Computational result of mathematical model 2

\begin{tabular}{cccc}
\hline$D_{\text {MAX }}$ & TC (MU) & MTD (DMU) & CPU time (s) \\
\hline & 3.5 & \multicolumn{3}{c}{ Infeasible } \\
4.0 & 1,577 & 3.905 & 0.03 \\
4.5 & 1,467 & 4.104 & 0.03 \\
5.0 & 1,324 & 4.866 & 0.03 \\
5.5 & 1,289 & 5.092 & 0.06 \\
6.0 & 1,289 & 5.092 & 0.09 \\
6.5 & 1,227 & 6.438 & 0.03 \\
7.0 & 1,227 & 6.860 & 0.08 \\
7.5 & 1,227 & 6.860 & 0.08 \\
8.0 & 1,225 & 7.728 & 0.06 \\
\hline
\end{tabular}

$D_{M A X}$ : maximum allowed average traveling distance, TC: total establishment cost, MTD: maximum travelling distance, MU: monetary unit, DMU: distance measurement unit.

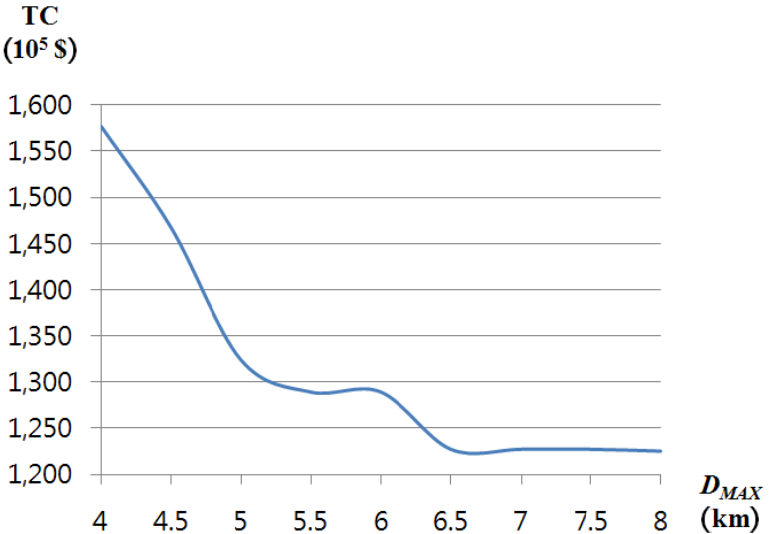

Figure 2. Relationship between total establishment cost (TC) and maximum allowed average traveling.

$D_{\text {MAX }}$ restrictions, a large amount of TC is required while it guarantees much convenience to patients. On the other hand, if we increase the value of $D_{M A X}$, it can save the budget at the expense of inconvenience of patients.

Therefore, at the formulation stage of government policy on the public long-term care facilities, inconvenience of patients needs to be considered against the economy of government budget.

\section{$3.3\left(S_{\text {Max }}, M_{\text {Max }}, L_{\text {Max }}\right)$ vs. Budget and ATDP}

Also, sensitivity analysis on $\left(S_{M a x}, M_{M a x}, L_{M a x}\right)$ was conducted. In order to focus on the effects of upper limits, budget and $D_{M A X}$ restrictions were relaxed by setting 3,000 MU for budget and $300 \mathrm{DMU}$ for $D_{M A X}$. Table 5 lists the results obtained from four different values of upper limits. Due to the nature of the objective function, model 1 tends to generate a large number of surpluses in capacity, resulting in inefficient use of budget. On the other hand, model 2 tries to build a smaller number of facilities provided that the patient demands are satisfied. And increase in $\left(S_{\operatorname{Max}}, M_{\operatorname{Max}}, L_{\operatorname{Max}}\right)$ has no effect beyond a certain set of values, i.e., $(7,7,7)$. With the second model we may achieve high utilization of facilities together with longer travel distance of patients. Thus it can be concluded that in making location and allocation decisions for long-term care facilities, an appropriate upper limit needs to be determined in advance to balance ATDP and the economic use of budget.

\subsection{Imputed Cost of Closest Assignment Rule}

Finally, the imputed cost of the closest assignment rule was estimated through comparison of the solutions of two models, model 2.1 and 2.2, obtained through slight modification of mode 12.Model 2.1 is formed by eliminating constraints (5) and (15) of model 2 while model 2.2 is obtained after eliminating constraint (15) only. With the capacity set of $(300,500,700)$ for $\left(C^{s}, C^{m}, C^{l}\right)$, the 
Table 5. Sensitivity analysis on $\left(S_{M a x}, M_{M a x}, L_{M a x}\right)$

\begin{tabular}{|c|c|c|c|c|c|c|c|c|}
\hline \multirow[b]{2}{*}{$\left(S_{\operatorname{Max}}, M_{M a x}, L_{M a x}\right)$} & \multicolumn{4}{|c|}{ Model 1} & \multicolumn{4}{|c|}{ Model 2} \\
\hline & $(6,6,6)$ & $(7,7,7)$ & $(8,8,8)$ & $(9,9,9)$ & $(6,6,6)$ & $(7,7,7)$ & $(8,8,8)$ & $(9,9,9)$ \\
\hline \multicolumn{9}{|l|}{ No. of facilities (unit) } \\
\hline Small & 6 & 7 & 8 & 7 & 6 & 7 & 7 & 7 \\
\hline Medium & 6 & 7 & 8 & 9 & 5 & 6 & 6 & 6 \\
\hline Large & 6 & 7 & 8 & 8 & 5 & 4 & 4 & 4 \\
\hline Surplus in capacity (person) & 1,984 & 3,484 & 4,984 & 5,184 & 784 & 884 & 884 & 884 \\
\hline
\end{tabular}

Table 6. Imputed cost of closest assignment rule

\begin{tabular}{|c|c|c|c|c|c|c|}
\hline \multirow{2}{*}{$\left(S_{M a x}, M_{M a x}, L_{M a x}\right)$} & \multicolumn{2}{|c|}{$\begin{array}{c}\text { Model } 2.2 \\
\text { (with closest assignment rule) }\end{array}$} & \multicolumn{2}{|c|}{$\begin{array}{c}\text { Model 2.1 } \\
\text { (without closest assignment rule) }\end{array}$} & \multirow{2}{*}{$\begin{array}{l}\text { Imputed cost } \\
\text { (MU) }\end{array}$} & \multirow{2}{*}{$\begin{array}{l}\text { Difference in } \\
\text { MTD (DMU }\end{array}$} \\
\hline & TC (MU) & MTD (DMU) & $\mathrm{TC}(\mathrm{MU})$ & MTD (DMU) & & \\
\hline$(6,6,6)$ & 1,152 & 12.928 & 1,013 & 35.930 & 139 & 23.002 \\
\hline$(7,7,7)$ & 1,149 & 11.910 & 1,007 & 46.166 & 142 & 34.256 \\
\hline$(8,8,8)$ & 1,149 & 13.030 & 1,007 & 46.166 & 142 & 33.136 \\
\hline$(9,9,9)$ & 1,149 & 11.910 & 1,004 & 37.834 & 145 & 25.924 \\
\hline$(10,10,10)$ & 1,149 & 13.030 & 1,004 & 36.060 & 145 & 23.030 \\
\hline
\end{tabular}

TC: total establishment cost, MTD: maximum travelling distance, MU: monetary unit, DMU: distance measurement unit.

computational results are shown at Table 6 .

We can state that the Seoul city government has to spend on the average $142.6 \mathrm{MU}$ or $14.16 \%$ of TC to keep closest assignment rule. However, the rule has the positive effects on MTD, i.e., decreases the value of MTD by 27.870 DMU or $68.93 \%$ of those under model 2.1 . Therefore, on the question of whether closest assignment rule would be implemented or not, it is advisable for city administrator to make prudent decision considering both the city's financial situation and patient's preference in terms of facility accessibility.

\section{CONCLUDING REMARKS}

An important social issue nowadays at national level is the preparation for an aging society. According to the National Health Insurance Corporation, as of 2009, only 59 public long-term care facilities have been set up by 48 provincial governments among 232 cities and provinces. For the Seoul metropolitan city with 25 boroughs, only 17 public facilities are currently in operation, which is substantially in short supply. In this study, through the development of mathematical models, we tried to determine adequate locations of the public long-term care facilities to be built to serve the patients with senile diseases. To reflect the preference of patients and their families, it was assumed that patients are assigned to a nearest established facility. Also, considering the differences in demands and economic situation in regions, we treated the size of facility as a decision variable. Two mathematical models were developed: one with the objective of minimizing the
ATDP under budget restriction and the other with the objective of minimizing the total opening costs under the restriction of maximum allowed travelling distance of patient group. CPLEX 12.1 was adopted to find the optimal solution of the developed models. Through sensitivity study, relationships among system parameters were investigated. We hope that the results in this study could provide references in formulating the location and allocation plan of the public long-term care facilities. As further studies, search for an exact solution method can be suggested including the branch and bound method. Also, the static nature of the proposed models could be extended to dynamic cases by considering the multi-period problem.

\section{ACKNOWLEDGMENTS}

This research was supported by Basic Science Research Program through the National Research Foundation of Korea funded by the Ministry of Education, Science and Technology (2012-009448).

\section{REFERENCES}

Adenso-Diaz, B. and Rodriguez, F. (1997), A simple search heuristic for the MCLP: application to the location of ambulance bases in a rural region, Omega, 25(2), 181-187.

Berman, O., Krass, D., and Wang, J. (2006), Locating service facilities to reduce lost demand, IIE Transactions, 38(11), 933-946. 
Daskin, M. S. and Dean, L. K. (2004), Location of healthcare facilities. In: Brandeau, M. L., Sainford, F., and Pierskalla, W. P. (eds), Operations Research and Health Care: A Handbook of Methods and Applications, Kluwer Academic, Boston, MA, chapter 3, 43-76.

Dearing, P. M. and Newruck, F. C. (1979), A capacitated bottleneck facility location problem, Management Science, 25(11), 1093-1104.

Jia, H., Ordonez, F., and Dessouky, M. (2007), A modeling framework for facility location of medical services for large-scale emergencies, IIE Transactions, 39(1), 41-55.

Kim, D. G. and Kim, Y. D. (2010), A branch and bound algorithm for determining locations of long-term care facilities, European Journal of Operational Research, 206(1), 168-177.

Kim, D. G., Kim, Y. D., and Lee, T. S. (2012), Heuristics for locating two types of public health-care facilities,
Industrial Engineering \& Management Systems, 11(2), 202-214.

Ndiaye, M. and Alfares, H. (2008), Modeling health care facility location for moving population groups, Computers \& Operations Research, 35(7), 2154-2161.

Nozick, L. K. (2001), The fixed charge facility location problem with coverage restrictions, Transportation Research Part E: Logistics and Transportation Review, 37(4), 281-296.

Rahman, S. and Smith, D. K. (2000), Use of locationallocation models in health service development planning in developing nations, European Journal of Operational Research, 123(3), 437-452.

Sankaran, J. K. and Raghavan, N. R. S. (1997), Locating and sizing plants for bottling propane in South India, Interfaces, 27(6), 1-15.

Verter, V. and Lapierre, S. D. (2002), Location of preventive health care facilities, Annals of Operations Research, 110(1-4), 123-132. 